\title{
A SMOOTH MODEL OF THE RESULTANT FRICTION FORCE ON A PLANE CONTACT AREA
}

\author{
Grzegorz Kudra, Jan Awrejcewicz \\ Lodz University of Technology, Department of Automation, Biomechanics and Mechatronics, Eódź, Poland \\ e-mail: grzegorz.kudra@p.lodz.pl
}

\begin{abstract}
A class of smooth approximations of the total friction force occurring on a plane finite contact surface is presented. It is assumed that the classical Coulomb friction law is valid on any infinitesimal element of the contact region. The models describe the stick phase, the fully developed sliding and the transition between these two modes. They take into account different values of static and dynamic friction coefficients. The models are applied in simulation of a dynamical system performing translational and rotational stick-slip oscillations, and then they are verified by comparison with the corresponding results in which an event-driven discontinuous model of friction is used.
\end{abstract}

Keywords: friction modelling, Coulomb-Contensou model, stick-slip, plane contact

\section{Introduction}

Contensou (1963) derived the integral model of resultant friction forces between two contacting bodies on the assumption of validity of the Coulomb friction law on each element of a circular contact area, Hertzian contact stresses and fully developed sliding. He pointed out that the normal component of relative angular velocity cannot be neglected in the model of friction forces for a certain class of mechanical systems.

Howe and Cutkosky (1996), for practical purposes of robotics, proposed an approximation of this problem in the form of ellipsoid description of the limit surface bounding the zone of admissible tangential loadings of the contact. However, it is not a direct description of the relation between the components of sliding and friction. Some researchers proposed Padé approximations as a convenient substitution of the integral model of friction forces (Zhuravlev, 1998; Zhuravlev and Kireenkov, 2005). Then the Padé approximants (Kireenkov, 2008) and hyperbolic tangent functions (Kudra and Awrejcewicz, 2011b) were used in the modelling of friction forces in the case of a circular contact zone and presence of rolling resistance. Kosenko and Aleksandrov (2009) developed a piece-wise linear approximation of the integral model of friction in the case of elliptical contact zone and Hertzian contact stresses. Kudra and Awrejcewicz (2012a, 2013) presented approximations which can be understood as a certain kind of generalizations and modifications of the Pade approximants, and applied them to the above problems including the case of the circular contact with uniform contact pressure distribution as well as elliptical one with the presence of rolling resistance.

The above mentioned models approximate the friction forces during the fully developed sliding or their limits in the stick mode. The simulation requires also models describing the transition between these two modes. The assumption of negligibly short time of duration of this transition results in a non-smooth dynamical system. They can often be modelled by the use of piecewise smooth differential equations (PWS). Then the PWS systems are sometimes divided in the following way (Leine and Nijmeijer, 2004): a) systems with a continuous but non-smooth 
vector field; b) systems with a continuous but non-smooth state; c) systems with a discontinuous state.

Existence and uniqueness of the solution are guaranteed only for systems belonging to group (a), known also as Filippov type systems (Filippov, 1964, 1988; Leine and Nijmeijer, 2004). For example, a mechanical system with dry friction modelled as a discontinuous function of time belongs to group (b). For instance, the assumption of rigidity of contacting bodies and the friction force dependent on normal load, which is not known in advance, can lead in some cases to inconsistency of the solution, known as the Painlevé paradox (Jellet, 1872; Painlevé, 1895; Génot and Brogliato, 1999). Such cases should be interpreted in such a way that the mathematical model and solution concept do not correspond to the real physical phenomenon.

The techniques of simulation of non-smooth systems can be divided in the following way (Leine and Nijmeijer, 2004): (i) event-driven integration methods; (ii) time-stepping methods. Acary and Brogliato (2010) presented a comprehensive review of the event-driven and timestepping numerical methods for simulation of non-smooth dynamical systems. Sometimes as another way of dealing with the non-smooth dynamical systems are regularization or smoothing methods.

Event driven methods (Leine and Nijmeijer, 2004) use classical integration methods between two successive events (switches between two different modes) when a system behaves smoothly. The integration stops if the event is detected. Then the system may undergo a step change and the next mode is determined. Special rules for the system change can be constructed in order to avoid inconsistency of the solution mentioned above. Kudra and Awrejcewicz (2012a) developed an event driven simulation algorithm of a mechanical system with circular frictional contact with a uniform contact pressure distribution and the Coulomb friction law. The scheme uses approximations of the integral friction model for both the fully developed sliding and stick phase (for determination of the end of the stick mode), but different values of static and (constant) dynamic friction coefficients are used. The combined translational and rotational stick-slip oscillations are presented.

Time-stepping methods are special numerical schemes which do not require detection of the events (Moreau, 1988; Stewart and Trinkle, 1996; Jean, 1999; Awrejcewicz and Lamarque, 2003). Some authors (Leine and Glocker, 2003; Möller et al., 2009) constructed such algorithms with appropriate approximations of the friction forces for a finite circular contact area with the Hertz stress distribution and Coulomb friction law on each element of the contact.

The regularization methods are modifications of the mathematical model leading to a lower degree of "non-smoothness" of the dynamical system. Particularly, they can result in a smooth dynamical system, allowing for the use of classical integration methods. In the case of dry friction modelling, a common approach is to replace the set-valued friction force in the stick mode by the force of action of a stiff spring with some saturation threshold, usually supplemented with additional damping elements or with additional state variables resulting in better description of the real phenomenon (Do et al., 2007). In the instance of a one-dimensional dry friction problem (i.e. either pure translational or rotational relative displacement of the contact), one can encounter smooth approximations of the friction force, where the signum function is replaced by such functions like arctangent or hyperbolic tangent. If this function is multiplied by another expression describing the changes of the dynamic friction coefficient during motion, then the phenomenon when the friction coefficient decreases at the beginning of the movement can be modelled (Awrejcewicz and Olejnik, 2003; Pilipchuk and Tan, 2004; Awrejcewicz et al., 2008).

The drawback of the above mentioned regularizations may be stiffness of the resulting ordinary differential equations. Sometimes they may change some properties of the model, especially may change properties or destroy the equilibrium related to the stick mode. Also, in some regularized models, the loos of stability of the equilibrium and occurrence of artificial oscillations is possibly. On the other hand, the regularizations in general decrease the risk of the occurrence of 
problems with the solution inconsistency. In particular, if the friction force is not a discontinuous function of sliding velocity (it may be a non-smooth function), the system belongs to the above mentioned group (a) and the solution uniqueness and existence are guaranteed. There are many works in which the influence of the friction model on the properties of the resulting mechanical system, including stability and bifurcations of the related solutions, is investigated (Bogacz and Sikora, 1990; Sikora and Bogacz, 1993; Leine et al., 2000).

In the case of combined translational and rotational relative displacement of a finite contact, there are smooth models developed in which singularities of the expressions are avoided by the addition of a small positive parameter in special places of approximations of the integral models (Kudra and Awrejcewicz, 2011b, 2012b). However, these constructions do not assume the possibility for the friction coefficient to decrease in the beginning of slip. Stamm and Fidlin (2007, 2008) developed a regularized two-dimensional model based on the elastic-plastic theory and being a generalization of similar approaches for one-dimensional contact. It requires, however, discretization of the contact area (in contrast to the other above-referred models for two-dimensional contact), leading to much higher computational cost.

In this paper, we present smooth approximations of the friction force and torque appearing between two bodies contacting on a plane contact area of an arbitrary shape. They are applied to a special mechanical system in which combined translational and rotational stick-slip oscillations appear and the results are compared to those obtained by the event-driven numerical algorithm (Kudra and Awrejcewicz, 2012a). A part of the present work was previously presented in a shortened version (Kudra and Awrejcewicz, 2011a).

\section{Integral model of friction}

Let us consider the plane contact area $F$ whose dimensionless form is presented in Fig. 1a. The Cartesian coordinate system Axyz is introduced, where the axes $x$ and $y$ lie in the contact plane. The dimensionless length is related to the real characteristic dimension of the contact $\widehat{a}$, therefore the dimensionless coordinates of the point situated on the contact plane are $x=\widehat{x} / \widehat{a}$ and $y=\widehat{y} / \widehat{a}$, where $\widehat{x}$ and $\widehat{y}$ are the corresponding real coordinates. The non-dimensional elementary friction force acting on the element $d F$ of the contact area is defined as $d \mathbf{T}=d \widehat{\mathbf{T}} /(\mu \widehat{N})$, where $d \widehat{\mathbf{T}}_{s}$ is its real counterpart, $\widehat{N}$ is the real normal component of the total force of interaction between two bodies, while $\mu$ is the dry friction coefficient during slip. The moment of the force $d \mathbf{T}$ about the point $A$ (center of contact) reads $d \mathbf{M}=\boldsymbol{\rho} \times d \mathbf{T}=d \widehat{\mathbf{M}} /(\widehat{a} \mu \widehat{N})$, where $d \widehat{\mathbf{M}}$ is the real counterpart of $d \mathbf{M}$. The dimensionless contact pressure distribution is defined as $\sigma(x, y)=\widehat{\sigma}(x, y) \widehat{a}^{2} / \widehat{N}$, where $\widehat{\sigma}(x, y)$ is the real pressure. For the purpose of compatibility with the dimensionless model of the mechanical system presented in Section 5, we additionally introduce the dimensionless time $t=\alpha \widehat{t}$, where $\widehat{t}$ denotes its real counterpart.

It is assumed that the contact $F$ can only operate in the two modes: the stick and the fully developed sliding (the transition between these two modes is infinitely short). During the sliding, local relative motion of the contacting bodies can be assumed as plane motion of the rigid body. Moreover, we assume that the spatial Coulomb friction law is valid on each element $d F$

$$
d \mathbf{T} \in \begin{cases}-\sigma(x, y) \frac{\mathbf{v}_{P}}{\left\|\mathbf{v}_{P}\right\|} d F & \text { for } \quad \mathbf{v}_{P} \neq \mathbf{0} \\ \left.\left\{\mathbf{u} \in \mathbb{R}^{2}: \| \mathbf{u}\right\} \leqslant \eta \sigma(x, y) d F\right\} & \text { for } \quad \mathbf{v}_{P}=\mathbf{0}\end{cases}
$$

where $\eta$ is the ratio of the static friction coefficient to the dynamic one, and $\mathbf{v}_{P}$ is velocity of the element $d F$ (see Fig. 1b).It is a priori assumed that the elementary friction force $d \mathbf{T}$ as well as the relative sliding velocity $\mathbf{v}_{P}$ lie in the plane of the contact $F$, so the problem described by Eq. (2.1) is in fact the two-dimensional one. 

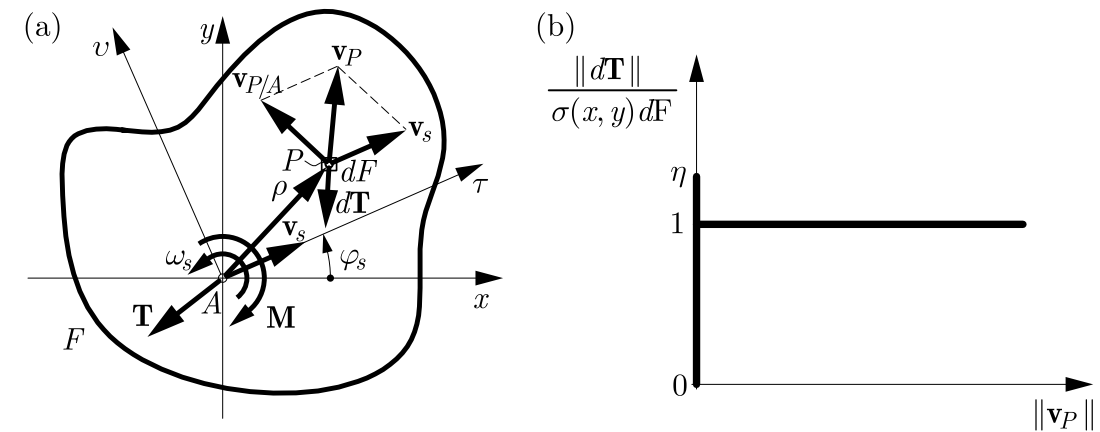

Fig. 1. The plane contact area (a) and the magnitude of the elementary friction force as a function of the magnitude of relative velocity (b)

The system of elementary friction forces can be reduced to the force $\mathbf{T}=T_{x} \mathbf{e}_{x}+T_{y} \mathbf{e}_{y}=$ $T_{\tau} \mathbf{e}_{\tau}+T_{v} \mathbf{e}_{v}$ acting at the point $A$ and the corresponding moment $\mathbf{M}=M \mathbf{e}_{z}$, where $\mathbf{e}_{x}, \mathbf{e}_{y}, \mathbf{e}_{z}$, $\mathbf{e}_{\tau}$ and $\mathbf{e}_{v}$ are the unit vectors of the corresponding axes.

During the slip mode, relative motion of the contact area is described by the use of the following quantities: $\mathbf{v}_{s}=\widehat{\mathbf{v}}_{s} /(\alpha \widehat{a})=v_{s x} \mathbf{e}_{x}+v_{s y} \mathbf{e}_{y}=v_{s} \mathbf{e}_{\tau}$-dimensionless linear velocity of the pole $A, \boldsymbol{\omega}_{s}=\widehat{\boldsymbol{\omega}}_{s} / \alpha=\omega_{s} \mathbf{e}_{z}$ - dimensionless angular velocity of the contact $\left(\widehat{\mathbf{v}}_{s}\right.$ and $\widehat{\boldsymbol{\omega}}_{s}$ denote the corresponding real counterparts). Then the components of the friction force and moment can be obtained by the use of the following integrals

$$
\begin{aligned}
& T_{x}=-T_{s x}\left(\theta_{s}, \varphi_{s}\right)=-\iint_{F} \sigma(x, y) \frac{\cos \theta_{s} \cos \varphi_{s}-y \sin \theta_{s}}{\sqrt{\left(\cos \theta_{s} \cos \varphi_{s}-y \sin \theta_{s}\right)^{2}+\left(\cos \theta_{s} \sin \varphi_{s}+x \sin \theta_{s}\right)^{2}}} d x d y \\
& T_{y}=-T_{s y}\left(\theta_{s}, \varphi_{s}\right)=-\iint_{F} \sigma(x, y) \frac{\cos \theta_{s} \cos \varphi_{s}+x \sin \theta_{s}}{\sqrt{\left(\cos \theta_{s} \cos \varphi_{s}-y \sin \theta_{s}\right)^{2}+\left(\cos \theta_{s} \sin \varphi_{s}+x \sin \theta_{s}\right)^{2}}} d x d y \\
& M=-M_{s}\left(\theta_{s}, \varphi_{s}\right)=-\iint_{F} \sigma(x, y) \frac{\left(x^{2}+y^{2}\right) \sin \theta_{s}+x \cos \theta_{s} \sin \varphi_{s}-y \cos \theta_{s} \cos \varphi_{s}}{\sqrt{\left(\cos \theta_{s} \cos \varphi_{s}-y \sin \theta_{s}\right)^{2}+\left(\cos \theta_{s} \sin \varphi_{s}+x \sin \theta_{s}\right)^{2}}} d x d y
\end{aligned}
$$

where the angles $\theta_{s}$ and $\varphi_{s}$ are defined in such a way, that

$$
\begin{array}{lll}
v_{s}=\lambda_{s} \cos \theta_{s} & \omega_{s}=\lambda_{s} \sin \theta_{s} & \lambda_{s}=\sqrt{v_{s}^{2}+\omega_{s}^{2}} \\
v_{s x}=v_{s} \cos \varphi_{s} & v_{s y}=v_{s} \sin \varphi_{s} &
\end{array}
$$

The signs of the functions $T_{s x}, T_{s y}$ and $M_{s}$ are changed in order to simplify the further notation.

Then the full model of the friction forces can be defined as

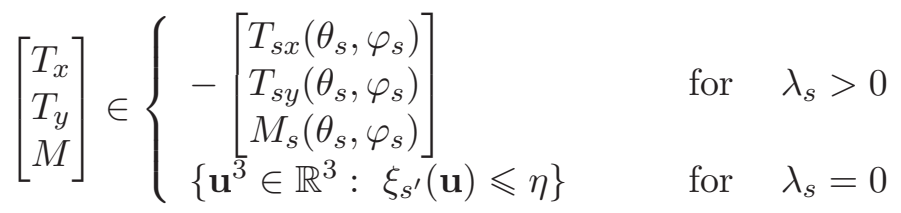

where the bottom part of the expression concerns the stick mode. In this case the scalar function $\xi_{s^{\prime}}(\mathbf{u}) \geqslant 0$ is defined as a part of the solution $\left(\xi_{s^{\prime}}, \theta_{s^{\prime}}, \varphi_{s^{\prime}}\right)$ to the following set of algebraic equations

$$
\mathbf{u}=-\xi_{s^{\prime}}\left[\begin{array}{c}
T_{s x}\left(\theta_{s^{\prime}}, \varphi_{s^{\prime}}\right) \\
T_{s y}\left(\theta_{s^{\prime}}, \varphi_{s^{\prime}}\right) \\
M_{s}\left(\theta_{s^{\prime}}, \varphi_{s^{\prime}}\right)
\end{array}\right]
$$


In Eq. (2.5), the angles $\theta_{s^{\prime}}$ and $\varphi_{s^{\prime}}$ can be interpreted in such a way that $\lambda_{s^{\prime}} \cos \theta_{s^{\prime}} \cos \varphi_{s^{\prime}}=v_{s^{\prime} x}$, $\lambda_{s^{\prime}} \cos \theta_{s^{\prime}} \sin \varphi_{s^{\prime}}=v_{s^{\prime} y}$ and $\lambda_{s^{\prime}} \sin \theta_{s^{\prime}}=\omega_{s^{\prime}}\left(\lambda_{s^{\prime}}>0\right)$ are the components of virtual (possible sliding) in the event that the friction coefficient is sufficiently small. The above construction of the part of the model concerning the stick phase is motivated by the fact that during the stick mode the components of the friction force and moment $\left(T_{x}, T_{y}, M\right)$ lie inside the zone bounded by a surface parametrically described by the functions $T_{x}=-\eta T_{s x}\left(\theta_{s}, \varphi_{s}\right), T_{y}=-\eta T_{s y}\left(\theta_{s}, \varphi_{s}\right)$ and $M=-\eta M_{s}\left(\theta_{s}, \varphi_{s}\right)$. Since this zone is convex, there always exists one solution to equations (2.4) for $\xi_{s^{\prime}} \geqslant 0$. The solution to Eq. (2.5) can be obtained numerically by the use of Newtons' method using a proper starting point. Then the first component of the solution $\left(\xi_{s^{\prime}}, \theta_{s^{\prime}}, \varphi_{s^{\prime}}\right)$ is used for detection of the possible end of the stick mode for $\xi_{s^{\prime}}(\mathbf{u})=\eta$. The two remaining components $\left(\theta_{s^{\prime}}, \varphi_{s^{\prime}}\right)$ define the direction of slip in the beginning of the possible sliding. The criterion $\xi_{s^{\prime}}(\mathbf{u})=\eta$ can also be interpreted as the yield surface.

\section{Approximations of the integral model of sliding friction}

An exact integral model of friction forces (2.2) is computationally expensive and inconvenient for use in numerical simulations. Kudra and Awrejcewicz (2013) proposed different families of its approximations. Some of them can be presented in the following form

$$
f^{\left(I_{n}\right)}\left(\theta_{s}, \varphi_{s}\right)=\frac{\sum_{i=0}^{n} a_{f, i} \cos ^{n-i} \theta_{s} \sin ^{i} \theta_{s}}{\left(\left|\cos \theta_{s}\right|^{m n}+b^{m}\left|\sin \theta_{s}\right|^{m n}\right)^{m^{-1}}}
$$

where $f=T_{s x}, T_{s y}, M_{s}$, and $n$ is the order of the approximation. For the assumed model of the contact pressure distribution $\sigma(x, y)$, the functions $a_{f, i}=a_{f, i}\left(\varphi_{s}, \operatorname{sgn}\left(\cos \theta_{s}\right), \operatorname{sgn}\left(\sin \theta_{s}\right)\right)$ (it occurs that for an even number $n$ they do not depend on signs of $\cos \theta_{s}$ and $\sin \theta_{s}$ ) are found in such a way, that the following conditions are fulfilled

$$
\begin{array}{ll}
\frac{\partial^{i} f^{\left(I_{n}\right)}}{\partial \cos ^{i} \theta_{s}}=\frac{\partial^{i} f}{\partial \cos ^{i} \theta_{s}} & \text { for } \quad \cos \theta_{s}=0 \quad \text { and } \quad i=0,1, \ldots, n_{1} \\
\frac{\partial^{i} f^{\left(I_{n}\right)}}{\partial \sin ^{i} \theta_{s}}=\frac{\partial^{i} f}{\partial \sin ^{i} \theta_{s}} & \text { for } \quad \sin \theta_{s}=0 \quad \text { and } \quad i=0,1, \ldots, n_{2}
\end{array}
$$

where $n_{1}+n_{2}=n-1$. The other constants $m \geqslant 0$ and $b \geqslant 0$ do not influence conditions (3.2) and they can be found in the process of optimization of fitting the approximate model to the integral components, or identified experimentally.

Taking into account relations (2.3), approximations (3.1) take the form as follows

$$
f^{\left(I_{n}\right)}\left(v_{s}, \omega_{s}, \varphi_{s}\right)=\frac{\sum_{i=0}^{n} a_{f, i} v_{s}^{n-i} \omega_{s}^{i}}{\left(\left|v_{s}\right|^{m n}+b^{m}\left|\omega_{s}\right|^{m n}\right)^{m^{-1}}}
$$

For $n_{1}=0, n_{2}=0(n=1)$, one gets the following form of the approximation

$$
\begin{aligned}
& T_{s x}^{\left(I_{0,0}\right)}=\frac{v_{s x}-b c_{0,1,1}^{(x, y)} \omega_{s}}{\left(\left|v_{s}\right|^{m}+b^{m}\left|\omega_{s}\right|^{m}\right)^{m^{-1}}} \quad T_{s y}^{\left(I_{0,0}\right)}=\frac{v_{s y}+b c_{1,0,1}^{(x, y)} \omega_{s}}{\left(\left|v_{s}\right|^{m}+b^{m}\left|\omega_{s}\right|^{m}\right)^{m^{-1}}} \\
& M_{s}^{\left(I_{0,0}\right)}=\frac{b c_{0,0,-1}^{(x, y)} \omega_{s}-c_{0,1,0}^{(x, y)} v_{s x}+c_{1,0,0}^{(x, y)} v_{s y}}{\left(\left|v_{s}\right|^{m}+b^{m}\left|\omega_{s}\right|^{m}\right)^{m^{-1}}}
\end{aligned}
$$


where

$$
c_{i, j, k}^{(x, y)}=\iint_{F} x^{i} y^{j}\left(x^{2}+y^{2}\right)^{-\frac{k}{2}} \sigma(x, y) d x d y
$$

and the alternative notation $f^{\left(I_{n 1, n 2}\right)}$ have been introduced.

For a circularly symmetric contact pressure distribution $T_{v}=0$, we use notation $T_{s}=T_{s \tau}=-T_{\tau}$. In this case, we can also write that $T_{s x}=T_{s} \cos \varphi_{s}$ and $T_{s y}=T_{s} \sin \varphi_{s}$. Now $c_{0,1,1}^{(x, y)}=c_{1,0,1}^{(x, y)}=c_{0,1,0}^{(x, y)}=c_{1,0,0}^{(x, y)}=0$, and approximations (3.4) take the following form

$$
T_{s}^{\left(I_{0,0}\right)}=\frac{v_{s}}{\left(\left|v_{s}\right|^{m}+b^{m}\left|\omega_{s}\right|^{m}\right)^{m^{-1}}} \quad M_{s}^{\left(I_{0,0}\right)}=\frac{b c_{0,0,-1}^{(x, y)} \omega_{s}}{\left(\left|v_{s}\right|^{m}+b^{m}\left|\omega_{s}\right|^{m}\right)^{m^{-1}}}
$$

where $T_{s}^{\left(I_{0,0}\right)}$ is the approximation of $T_{s}\left(T_{s x}^{\left(I_{0,0}\right)}=T_{s}^{\left(I_{0,0}\right)} \cos \varphi_{s}\right.$ and $\left.T_{s y}^{\left(I_{0,0}\right)}=T_{s}^{\left(I_{0,0}\right)} \sin \varphi_{s}\right)$.

\section{Regularized model}

In the special case of $\omega_{s}=0$ or $\widehat{a}=0, \operatorname{models}(2.2)$ and (3.3)-(3.5) are $T_{s}=T_{s}^{\left(I_{n}\right)}=\operatorname{sgn} v_{s}$ $\left(T_{s}=-T_{\tau}, T_{v}=0, M_{s}=M_{s}^{\left(I_{n}\right)}=0\right)$. For the purpose of numerical simulations, often the regularization of the sign function is performed by the use of arctan or tanh functions (Awrejcewicz and Lamarque, 2003; Awrejcewicz and Olejnik, 2003; Kudra and Awrejcewicz, 2011b). We propose another way of regularization of the sign function

$$
T_{s \varepsilon}=T_{s \varepsilon}^{\left(I_{n}\right)}=\frac{v_{s}}{\sqrt{v_{s}^{2}+\varepsilon^{2}}}
$$

where $\varepsilon$ is a small numerical parameter.

In order to model the stick-slip oscillations, when the static friction coefficient is higher than the corresponding coefficient during the slip, one can develop model (4.1) in the following way

$$
T_{s \varepsilon_{2}}=T_{s \varepsilon_{2}}^{\left(I_{n}\right)}=v_{s}\left(\frac{1}{\sqrt{v_{s}^{2}+\varepsilon^{2}}}+\eta^{\prime} \frac{\varepsilon^{3}}{\left(v_{s}^{2}+\varepsilon^{2}\right)^{2}}\right)
$$

where the parameter $\eta^{\prime}$ is a certain function of the coefficient $\eta=\max \left|T_{s \varepsilon_{2}}\right|$. The function $\eta^{\prime}=$ $\eta^{\prime}(\eta)$ does not depend on $\varepsilon$ and can be approximated as $\eta^{\prime} \approx-13.607+30.893 \eta-22.01 \eta^{2}+5.878 \eta^{3}$ for $\eta \in[1,1.3]$ and $\eta^{\prime} \approx-2.41+3.985 \eta-0.3581 \eta^{2}+0.0493 \eta^{3}$ for $\eta \in[1.3,2.7]$, where the error is $|\Delta \eta|<0.001$. Figure 2 exhibits the exemplary plots of model (4.2).

Trying to generalize the above results and applying them to model (3.3), we assume in what follows

$$
f_{\varepsilon_{2}}^{\left(I_{n}\right)}\left(v_{s}, \omega_{s}, \varphi_{s}\right)=\sum_{i=0}^{n} a_{f, i} v_{s}^{n-i} \omega_{s}^{i}\left(\frac{1}{\sqrt{\lambda_{s b}^{2}+\varepsilon^{2}}}+\eta^{\prime} \frac{\varepsilon^{3}}{\left(\lambda_{s b}^{2}+\varepsilon^{2}\right)^{2}}\right)
$$

where

$$
\lambda_{s b}=\left(\left|v_{s}\right|^{m n}+b^{m}\left|\omega_{s}\right|^{m n}\right)^{m^{-1}}
$$

In the special case of $n=1\left(n_{1}=0, n_{2}=0\right.$, see Eq. (3.4)), one gets

$$
\begin{aligned}
& T_{s x \varepsilon_{2}}^{\left(I_{0,0}\right)}=\left(v_{s x}-b, c_{0,1,1}^{(x, y)} \omega_{s}\right) \lambda_{s b \varepsilon_{2}}^{(1)} \quad T_{s y \varepsilon_{2}}^{\left(I_{0,0}\right)}=\left(v_{s y}+b c_{1,0,1}^{(x, y)} \omega_{s}\right) \lambda_{s b \varepsilon_{2}}^{(1)} \\
& M_{s \varepsilon_{2}, 0}^{\left(I_{0,0}\right)}=\left(b c_{0,0,-1}^{(x, y)} \omega_{s}-c_{0,1,0}^{(x, y)} v_{s x}+c_{1,0,0}^{(x, y)} v_{s y}\right) \lambda_{s b \varepsilon_{2}}^{(1)}
\end{aligned}
$$




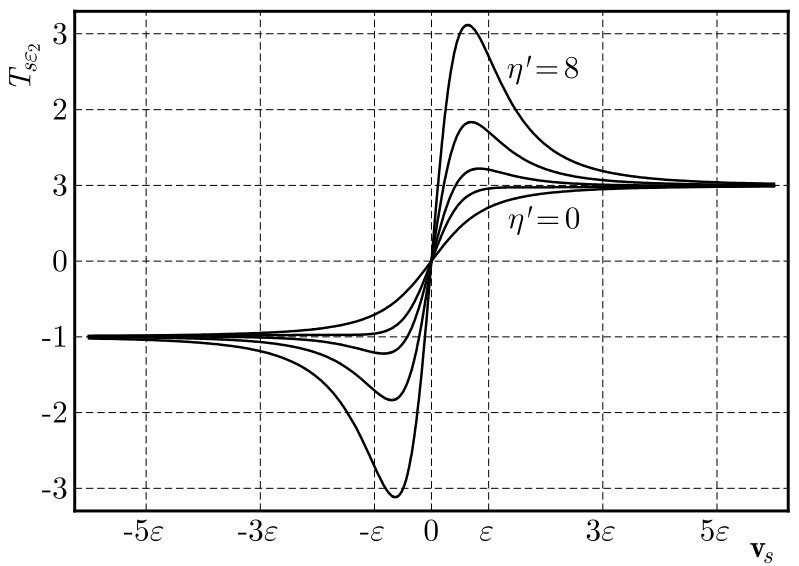

Fig. 2. The regularized model of friction (4.2) for $\eta^{\prime}=0, \eta^{\prime}=1, \eta^{\prime}=2, \eta^{\prime}=4$ and $\eta^{\prime}=8$

where

$$
\lambda_{s b \varepsilon_{2}}^{(1)}=\frac{1}{\sqrt{\left(\left|v_{s}\right|^{m}+b^{m}\left|\omega_{s}\right|^{m}\right)^{\frac{2}{m}}+\varepsilon^{2}}}+\eta^{\prime} \frac{\varepsilon^{3}}{\left(\left(\left|v_{s}\right|^{m}+b^{m}\left|\omega_{s}\right|^{m}\right)^{\frac{2}{m}}+\varepsilon^{2}\right)^{2}}
$$

For a circularly symmetric contact pressure distribution (see Eq. (3.5)) and $m=2$, relations (4.4) take the following form

$$
\begin{aligned}
& T_{s \varepsilon_{2}}^{\left(I_{0,0}\right)}=v_{s}\left(\frac{1}{\sqrt{v_{s}^{2}+b^{2} \omega_{s}^{2}+\varepsilon^{2}}}+\eta^{\prime} \frac{\varepsilon^{3}}{\left(v_{s}^{2}+b^{2} \omega_{s}^{2}+\varepsilon^{2}\right)^{2}}\right) \\
& M_{s \varepsilon_{2}}^{\left(I_{0,0}\right)}=b c_{0,0,-1}^{(x, y)} \omega_{s}\left(\frac{1}{\sqrt{v_{s}^{2}+b^{2} \omega_{s}^{2}+\varepsilon^{2}}}+\eta^{\prime} \frac{\varepsilon^{3}}{\left(v_{s}^{2}+b^{2} \omega_{s}^{2}+\varepsilon^{2}\right)^{2}}\right)
\end{aligned}
$$

\section{Example of application}

Here we recall the model of a mechanical system described and analysed in the work by Kudra and Awrejcewicz (2012a). The system, shown in Fig. 3, consists of a disk of radius $\widehat{r}=\widehat{a}$, mass $\widehat{m}$ and moment of inertia $\widehat{B}$. The disk is situated on a moving belt of velocity $\widehat{v}_{b}$. The position of the disk is defined by two co-ordinates: $\widehat{x}$ and $\varphi$ describing the linear and angular positions, respectively. The disk is joined with the support by the use of four elasto-damping cords winding the disk as shown in Fig. 3, where $\widehat{k}_{1} / 2, \widehat{k}_{2} / 2, \widehat{c}_{1} / 2$ and $\widehat{c}_{2} / 2$ are the corresponding coefficients of stiffness and damping.

It is assumed that the contact pressure distribution is circularly symmetric and $\widehat{\mathbf{T}}=\mu \widehat{N} T \mathbf{e}_{\tau}$ $\left(T=T_{\tau}=T_{x}, T_{v}=T_{y}=0\right)$ as well as $\widehat{\mathbf{M}}=\mu \widehat{N} \widehat{r} M \mathbf{e}_{z}$. Dynamics of the system is then governed by the following non-dimensional equations

$$
\left[\begin{array}{cc}
1 & 0 \\
0 & m
\end{array}\right]\left\{\begin{array}{l}
\ddot{x} \\
\ddot{\varphi}
\end{array}\right\}+\left[\begin{array}{cc}
c & c_{12} \\
c_{12} & c
\end{array}\right]\left\{\begin{array}{l}
\dot{x} \\
\dot{\varphi}
\end{array}\right\}+\left[\begin{array}{cc}
1 & k_{12} \\
k_{12} & 1
\end{array}\right]\left\{\begin{array}{l}
x \\
\varphi
\end{array}\right\}=\left\{\begin{array}{l}
\Gamma \\
\Omega
\end{array}\right\}
$$

where the symbol $(\bullet)$ stands for derivative with respect to the non-dimensional time $t$. 


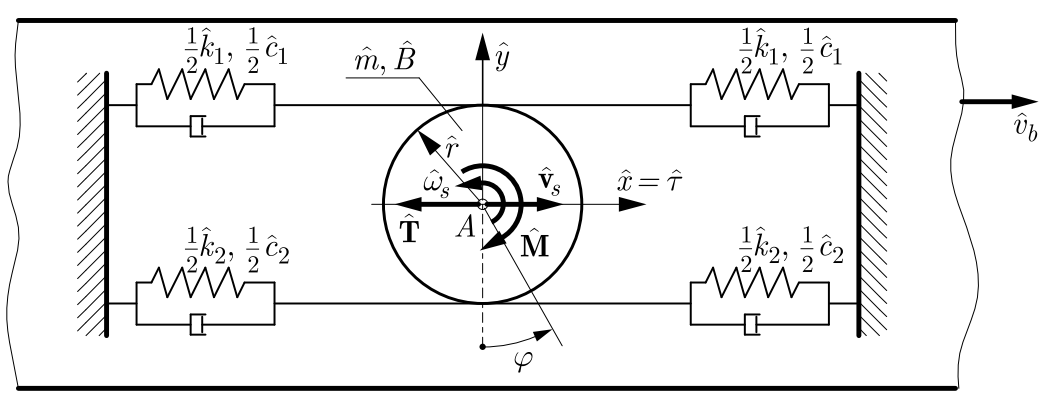

Fig. 3. The investigated mechanical system

The dimensionless variables used in Eq. (5.1) are defined in the following way

$$
\begin{array}{crrr}
x=\frac{\widehat{x}}{\widehat{r}} \quad m=\frac{\widehat{B}}{\widehat{m} \widehat{r}^{2}} & k_{12}=\frac{\widehat{k}_{2}-\widehat{k}_{1}}{\widehat{k}_{1}+\widehat{k}_{2}} & \Gamma=\bar{\mu} T & \Omega=\bar{\mu} M \\
c=\frac{\widehat{c}_{1}+\widehat{c}_{2}}{\sqrt{\widehat{m}\left(\widehat{k}_{1}+\widehat{k}_{2}\right)}} & c_{12}=\frac{\widehat{c}_{2}-\widehat{c}_{1}}{\sqrt{\widehat{m}\left(\widehat{k}_{1}+\widehat{k}_{2}\right)}} & v_{b}=\frac{\widehat{v}_{b}}{\alpha \widehat{r}} & t=\alpha \widehat{t}
\end{array}
$$

where

$$
\alpha=\sqrt{\frac{\widehat{k}_{1}+\widehat{k}_{2}}{\widehat{m}}} \quad \bar{\mu}=\frac{\mu \widehat{m} g}{\widehat{r}\left(\widehat{k}_{1}+\widehat{k}_{2}\right)}
$$

and where $\Gamma$ and $\Omega$ are the non-dimensional friction force and moment, respectively. Since $v_{s}=\alpha^{-1} \widehat{r}^{-1} \widehat{v}_{s}$ and $\omega_{s}=\alpha^{-1} \widehat{\omega}_{s}$, we get $v_{s}=\dot{x}-v_{b}$ and $\omega_{s}=\dot{\varphi}$, where $\dot{x}$ and $\dot{\varphi}$ are the dimensionless velocities of the disk.

In the work by Kudra and Awrejcewicz (2012a), a special event-driven scheme for numerical solution of equations (5.1) with the set-valued elements $\Gamma$ and $\Omega$ was developed. The solution is composed of segments obtained by the use of classical integration methods for stick or sliding modes. Each segment starts and ends with the events, i.e. switches between the two successive modes which are detected during simulation. The integral model of friction used for simulation of the sliding mode as well as detection of the end of the stick mode (see Section 2) is approximated by the use of Eq. (3.5) for $c_{0,0,-1}^{(x, y)}=2 / 3$ (uniform contact pressure distribution), $m=2$ and $b=1$.

In the present work, we test simulation of the above presented dynamical system using the regularized model of friction (4.5). Figure 4 exhibits two examples of periodic stick-slip attractors obtained for the following parameters of the system: $m=90, k_{12}=0.85, c=10^{-4}$, $c_{12}=0, v_{b}=0.15, \bar{\mu}=5$. The friction coefficient during the stick mode is defined by the use of $\eta=4.98\left(\eta^{\prime}=13.7627\right)$ for the first attractor (a)-(b) and $\eta=2.7\left(\eta^{\prime}=6.7627\right)$ for the second orbit (c)-(d). Each attractor is obtained two times through the event-driven scheme (Kudra and Awrejcewicz, 2012a) and presented in the current work as a regularized model, and then plotted two times. Since there is no visible difference between them (the plots cover each other), one can conclude that the proposed smooth model of friction works correctly. In the event-driven numerical algorithm, the threshold of detection of the singularity $\lambda_{s}=0$ during the sliding mode equals $10^{-7}$. For integration of the differential equations between the two successive events, we use the explicit Runge-Kutta (4,5) formula (Dormand-Prince) with relative and absolute tolerances equal to $10^{-10}$. In the case of integration of stiff differential equations with the regularized friction model, in which it is assumed $\varepsilon=10^{-5}$, we use a multistep implicit scheme based on numerical differential formulas with relative and absolute tolerances set to $10^{-10}$. The value of the parameter $\varepsilon$ should be chosen based on numerical experiments. 
(a)

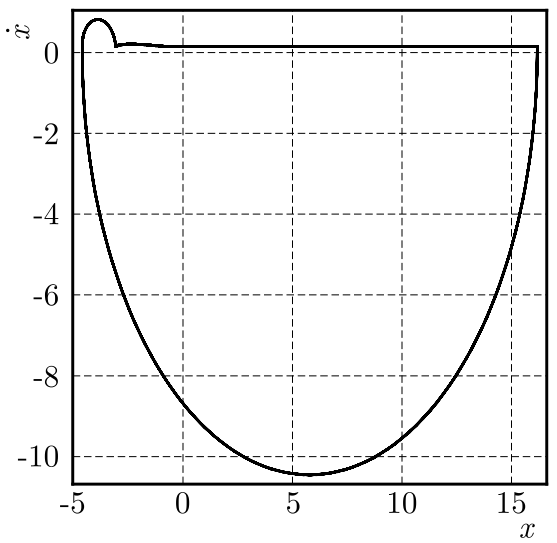

(c)

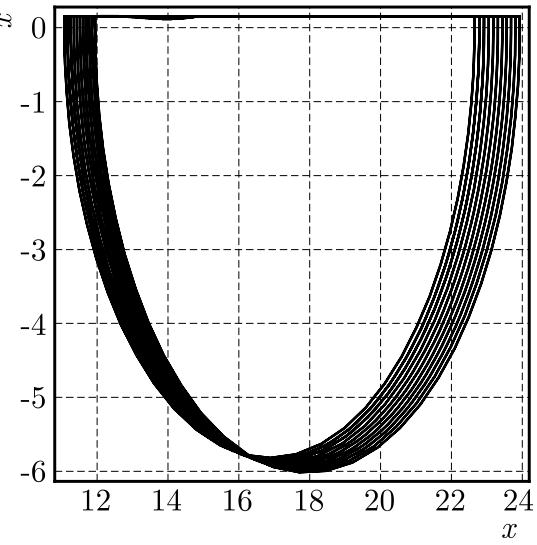

(b)

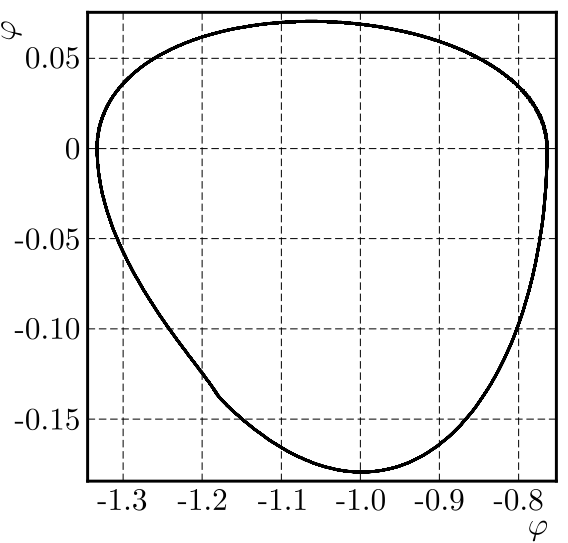

(d)

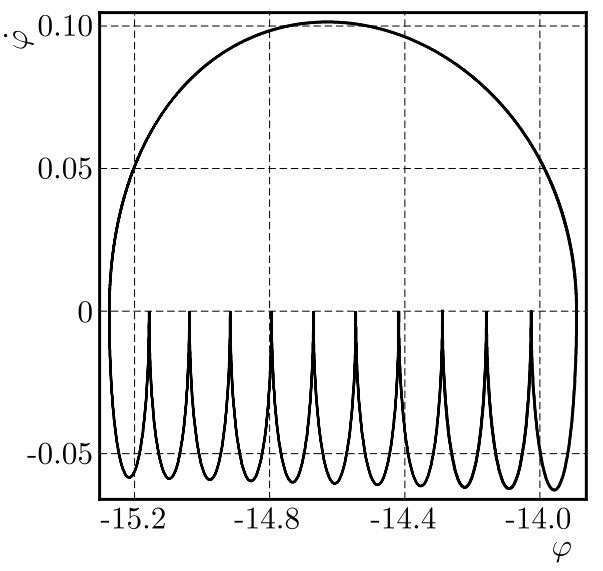

Fig. 4. Two examples of periodic attractors for $\eta=4.98$ (a)-(b) and $\eta=2.7$ (c)-(d) obtained from two different methods: event-driven scheme (Kudra and Awrejcewicz, 2012a) and regularization of the friction model

\section{Concluding remarks}

In the work, a new kind of regularized approximate model of the coupled friction force and torque for the general plane contact has been presented and tested. The developed here model approximates the "exact" integral model in which the classical Coulomb friction law on each element of the contact area and sudden switches between the stick mode and the fully developed sliding are assumed.

The main properties of the new model are: i) avoidance of the problem of time consuming integration over the contact area at each time step of integration of the differential equations, ii) avoidance of singularity problems or set-valued friction forces in the original model of friction and possibility of the use of classical algorithms for integration of the differential equations, iii) possibility to model static friction higher than the sliding one and stick-slip oscillations, iv) parameters of the approximate model can be found based on optimization of the fitting to the integral model or can be identified experimentally (independently of the integral friction model). The above pointed out properties can be understood as advantages. The new model possesses also a drawback, i.e. it makes the resulting differential equations stiff and thus requires special numerical methods.

The proposed model of friction has been applied to the modelling and simulation of a special mechanical system in which two-dimensional (linear and rotational) stick slip oscillation occur. The comparison of the results with those obtained by the event-driven method leads to the conclusion that the new model operates correctly. 
Acknowledgments

This work has been supported by the Polish National Science Centre, MAESTRO 2, No. 2012/04/A/ST8/00738. A part of this work was presented at the 11th Conference on Dynamical SystemsTheory and Applications, December 5-8, 2011, Łódź, Poland.

\section{References}

1. Acary V., Brogliato, B., 2010, Numerical Methods for Nonsmooth Dynamical Systems, Springer

2. Awrejcewicz J., Lamarque C.-H., 2003, Bifurcation and Chaos in Nonsmooth Mechanical Systems, World Scientific, Singapore

3. Awrejcewicz J., Olejnik P., 2003, Stick-slip dynamics of a two-degree-of-freedom system, International Journal of Bifurcation and Chaos, 13, 4, 843-861

4. Awrejcewicz J., Supę B., Lamarque C.-H., Kudra G., Wasilewski G., Olejnik P., 2008, Numerical and experimental study of regular and chaotic motion of triple physical pendulum, International Journal of Bifurcation and Chaos, 18, 10, 2883-2915

5. Bogacz, R., Sikora, J., 1990, On stability of periodic solutions of a discrete system with many degrees of freedom and dry friction, Proceedings of the Vibrations in Physical Systems, 57-59, Poznan, Poland

6. Contensou P., 1963, Couplage entre frottement de glissement et de pivotement dans la théorie de la toupe, [In:] Kreiselprobleme Gyrodynamic, Ziegler H. (Ed.), IUTAM Symposium, Celerina, 1962, Springer-Verlag, Berlin, 201-216

7. Do N.B., Ferri A.A., Bauchau O.A., 2007, Efficient simulation of a dynamic system with LuGre friction, Journal of Computational and Nonlinear Dynamics, 2, 4, 281-289

8. Filippov A.F., 1964, Differential equations with discontinuous right-hand side, American Mathematical Society Translations, 42, 199-231

9. Filippov A.F., 1988, Differential Equations with Discontinuous Right-Hand Sides, Kluwer Academic, Dordrecht

10. Génot F., Brogliato B., 1999, New results on Painlevé Paradoxes, European Journal of Mechanics - A/Solids, 18, 653-677

11. Howe R.D., Cutkosky M.R., 1996, Practical force-motion models for sliding manipulation, International Journal of Robotics Research, 15, 6, 557-572

12. JEAn M., 1999, The non-smooth contact dynamics method, Computer Methods in Applied Mechanics and Engineering, 177, 235-257

13. Jellet J.H., 1872, Treatise on the Theory of Friction, Hodges, Foster and Co, Dublin

14. Kireenkov A.A., 2008, Combined model of sliding and rolling friction in dynamics of bodies on a rough plane, Mechanicsof Solids, 43, 3, 412-425

15. Kosenko I., Aleksandrov E., 2009, Implementation of the Contensou-Erisman model of friction in frame of the Hertz contact problem on Modelica, 7th Modelica Conference, Como, Italy, 288-298

16. Kudra G., Awrejcewicz J., 2011a, Regularized model of coupled friction force and torque for circularly-symmetric contact pressure distribution, Proceedings of the 11th Conference on Dynamical Systems - Theory and Applications, 353-358

17. Kudra G., Awrejcewicz J., 2011b, Tangenshyperbolicus approximations of the spatial model of friction coupled with rolling resistance, International Journal of Bifurcation and Chaos, 21, 10, $2905-2917$

18. Kudra G., Awrejcewicz J., 2012a, Bifurcational dynamics of a two-dimensional stick-slip system, Differential Equations and Dynamical Systems, 20, 3, 301-322 
19. Kudra G., Awrejcewicz J., 2012b, Celtic stone dynamics revisited using dry friction and rolling resistance, Shock and Vibration, 19, 5, 1115-1123

20. Kudra G., Awrejcewicz J., 2013, Approximate modelling of resulting dry friction forces and rolling resistance for elliptic contact shape, European Journal of Mechanics - A/Solids, 42, 358-375

21. Leine R.I., van Campen D.H., Van De VRande B.L., 2000, Bifurcations in nonlinear discontinuous systems, Nonlinear Dynamics, 23, 2, 105-164

22. Leine R.I., Glocker Ch., 2003, A set-valued force law for spatial Coulomb-Contensou friction, European Journal of Mechanics - A/Solids, 22, 2, 193-216

23. Leine R.I., Nijmeijer H., 2004, Dynamics and Bifurcations of Non-smooth Mechanical Systems, Springer

24. Moreau J.J., 1988, Unilateral contact and dry friction in finite freedom dynamics, Nonsmooth Mechanics and Applications, 1-82, Springer-Verlag, Wien

25. Möller M., Leine R.I., Glocker Ch., 2009, An efficient approximation of orthotropic setvalued laws of normal cone type, Proceedings of the 7th EUROMECH Solid Mechanics Conference, Lisbon, Portugal

26. Painlevé P., 1895, Leçon sur le frottement, Hermann, Paris

27. Pilipchuk V.N., Tan C.A., 2004, Creep-slip capture as a possible source of squeal during decelerated sliding, Nonlinear Dynamics, 35, 259-285

28. Sikora, J., Bogacz, R., 1993, On dynamics of several degrees of freedom system, ZAMM Journal of Applied Mathematics and Mechanics, 73, T118-T122

29. Stamm W., Fidlin A., 2007, Regularization of 2D frictional contacts for rigid body dynamics, IUTAM Symposium on Multiscale Problems in Multibody System Contacts, 291-300, Springer

30. Stamm W., Fidlin A., 2008, Radial dynamics of rigid friction disks with alternating sticking and sliding, Proceedings of the 6th EUROMECH Nonlinear Dynamics Conference, Saint Perersburg

31. Stewart D., Trinkle J.C., 1996, An implicit time-stepping scheme for rigid body dynamics with inelastic collisions and Coulomb friction, International Journal of Numerical Methods in Engineering, 39, 2673-2691

32. Zhuravlev V.P., 1998, The model of dry friction in the problem of the rolling of rigid bodies, Journal of Applied Mathematics and Mechanics, 62, 5, 705-710

33. Zhuravlev V.P., Kireenkov A.A., 2005, Padé expansions in the two-dimensional model of Coulomb friction, Mechanics of Solids, 40, 2, 1-10 